\title{
High Security using Palm Vein Recognition Technology
}

\author{
Lakshmi Sudha.K \\ Research Scholar \\ Sathyabama University \\ SIES GST \\ Navi Mumbai, India
}

\author{
Neha Bhanushali \\ Department of IT \\ SIES GST \\ Navi Mumbai, India
}

\author{
Pooja Nikam \\ Department of IT \\ SIES GST \\ Navi Mumbai, India
}

\author{
Priti Tripathi \\ Department of IT \\ SIES GST \\ Navi Mumbai, India
}

\begin{abstract}
Palm Vein Recognition (PVR) technology uses a nearinfrared light to capture a user's palm vein pattern, generating a unique biometric template that is matched against preregistered users' palm vein patterns. It is a contactless authentication technology that offers an easy-to-use, hygienic solution to verify identity. This advanced, vascular pattern recognition solution is highly reliable and in a form factor that is non-intrusive and generates extremely fast authentication. The PVR device connects via USB to any PC or laptop. The interface included enables users to enjoy the simplicity associated with functionality while adding robust biometric authentication to enhance Security and protect mission-critical information and systems
\end{abstract}

\section{General Terms}

Biometrics, Pattern Recognition

\section{Keywords}

Region of Interest, Vein Bio-metrics, Segmentation, Thresholding, Imposter Attacks, Image Thinning

\section{INTRODUCTION}

In the ubiquitous network society, where individuals can easily access their information anytime and anywhere, people are also faced with the risk that others can easily access the same information anytime and anywhere. Because of this risk, personal identification technology, which can distinguish between registered legitimate users and imposters, is now generating interest .Currently, passwords, Personal Identification Numbers (4-digit PIN numbers) or identification cards are used for personal identification. However, cards can be stolen, and passwords and numbers can be guessed or forgotten. To solve these problems, biometric authentication technology, which identifies people by their unique biological information, is attracting attention [1]. In biometric authentication, an account holder's body characteristics or behaviors (habits) are registered in a database and then compared with others who may try to access that account to see if the attempt is legitimate. Fujitsu [11] is researching and developing biometric authentication technology focusing on four methods: fingerprints, faces, voiceprints, and palm veins. Among these, because of its high accuracy, contactless palm vein authentication technology is being incorporated into various financial solution products for use in public places. This paper introduces palm vein authentication technology and some examples of its application to financial solutions. It then describes Palm.
Secure, an authentication product that Fujitsu has developed for the general market and the company's key milestones in an effort to standardize Palm Secure for biometric authentication.

\section{EXISTING SYSTEM}

\subsection{Fingerprint Authentication}

Each fingerprint contains ridges in the skin which are detectable. These ridges make patterns which you can see with the naked eye. The ridge patterns can make loops, arches or whorls. In each fingerprint there are areas where one ridge changes, these points of change are called minutia. When a ridge ends, splits into two ridge, joins another ridge or creates a island; all of these features in a fingerprint are minutia.[2][4]

A fingerprint scanner detects the larger ridge patterns and records the following information about the minutia:

i) Orientation - the direction the minutia is facing.

ii) Spatial Frequency - how far apart specific features are located.

iii) Curvature - the rate of orientation change.

iv) Position - the $(\mathrm{x}, \mathrm{y})$ location relative to some fixed point

\section{Advantages -}

- Very high accuracy.

- Is the most economical biometric PC user authentication technique.

- It is one of the most developed biometrics.

- Easy to use.

- Small storage space required for the biometric template, reducing the size of the database memory required

- It is standardized.

\section{Disadvantages -}

- Uniqueness -

An important aspect of any biometric is that the characteristic be unique among all participants. Empirical evidence of fingerprints gathered since at least 1892, have found no two pairs of fingerprints that are identical, even between twins. While this is reassuring, more recent research indicates that the minutiae-based uniqueness as measured by fingerprint scanners may not be sufficient to determine individuality in every case.[2]

- For some people it is very intrusive, because is still related to criminal identification. 
- It can make mistakes with the dryness or dirty of the fingers skin, as well as with the age (is not appropriate with children, because the size of their fingerprint changes quickly).

\section{Limitations -}

- Fingerprint can be changed or damaged and some simply don't have fingerprints.

- Fingerprints are not very private, we leave fingerprints everywhere.

- Storing large amounts of fingerprint data in a safe way becomes a real challenge.

- If that data were exposed, criminals might devise a way to create fingerprints from the template data that fool fingerprint scanners.

\section{PROPOSED SYSTEM}

The Palm recognition system consists of an automatic segmentation system and is able to localize the palm vein regions. You have to store an palm vein image of an individual in database. Using that stored template in database we will be matching the present image. Based on the result we will be identifying a particular person. Along with palm vein identification system we will be designing a encryption/ decryption toolkit using palm vein code as key. The encryption and decryption process works in combination with a key - a word, number, or phrase - to encrypt the data. The same data encrypts to different cipher data with different keys.

The security of encrypted data is entirely dependent on two things:

1. The strength of the cryptographic algorithm.

2. The secrecy of the key.

But when we talk about the secrecy of the key then, in the case of passkey used as, a word, phrase or a number, any of these passkeys can be leaked out. Hence a better authentication technique is needed to be implemented so that it would help in the following-

1. No leakage of passkey.

2. High security to a confidential data.

3. Unique authentication for data.

So palm vein scanning is really a reliable means of identification. Unlike other forms of identification, such as passwords or keys, a person's iris cannot be stolen, forgotten or lost.

\subsection{Algorithms}

Three Algorithms are used:

\subsubsection{Vascular Pattern Marker Algorithm (VPMA)}

i. Open an Infrared Palm Image File in input mode.

ii. Convert the Loaded Image into Planar Image.

iii. The operator consists of a pair of $3 \times 3$ convolution kernels as shown in Figure below. One kernel is simply the other rotated by $90^{\circ}$.

\begin{tabular}{|l|l|l|}
\hline-1 & 0 & +1 \\
\hline-2 & 0 & +2 \\
\hline-1 & 0 & +1 \\
\hline
\end{tabular}

Gx

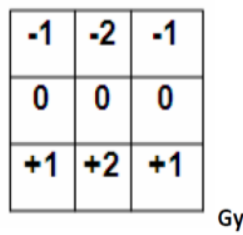

iv. Generated Planar Image in Step-ii, is passed through kernels created in Step iii.

v. Modified fine-grained Planar Image is stored into another Grayscale Image.

These kernels are designed to respond maximally to edges running vertically and horizontally relative to the pixel grid, one kernel for each of the two perpendicular orientations. The kernels can be applied separately to the input image, to produce separate measurements of the gradient component in each orientation (call these Gx and Gy). These can then be combined together to find the absolute magnitude of the gradient at each point and the orientation of that gradient. The gradient magnitude is given by:

$|\mathbf{G}|=\sqrt{ }(\mathbf{G x} \mathbf{2}+\mathbf{G y} \mathbf{2})$

Typically, an approximate magnitude is computed using:

$|\mathbf{G}|=|\mathbf{G x}|+|\mathbf{G y}|$

This is much faster to compute. The angle of orientation of the edge (relative to the pixel grid) giving rise to the spatial gradient is given by:

$\mathbf{q}=\arctan (\mathbf{G y} / \mathbf{G x})[3]$

\subsubsection{Vascular Pattern Extractor Algorithm}

(VPEA)

A digital grayscale image can be represented as a matrix of corresponding pixel values. A pixel is a small block that represents the amount of gray intensity to be displayed for that particular portion of the image. For most integers the pixel integers values range from 0 (Black) to 255 (White) [10]. The 256 possible gray intensity values are shown in Figure below

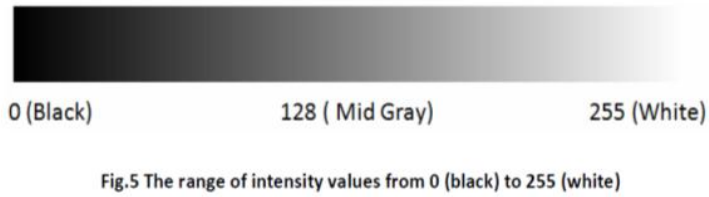

Using Vascular Pattern Extractor Algorithm, we get a grayscale image. We assume the resultant grayscale image file as gray.jpeg. Using the Vas_Pat_GBC_Algo we convert this gray.jpeg to a binary scale image as bin.jpeg [3]

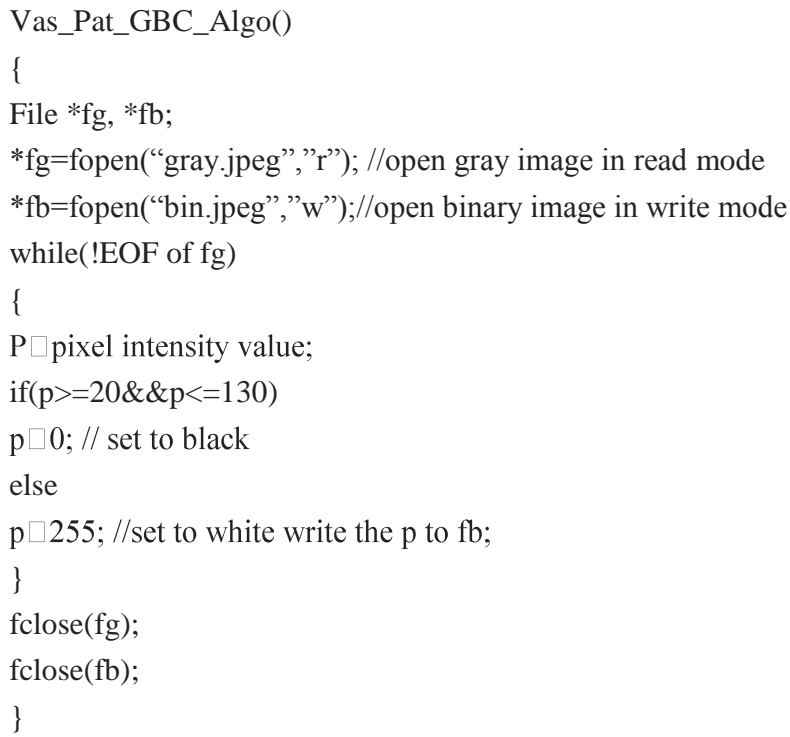




\subsubsection{Vascular Pattern Thinning Algorithm (VPTA)}

The proposed following algorithm is used for converting the 'bin.jpeg' into the 'thin.jpeg'. This 'thin.jpeg' file provides us a thinned vascular pattern which in turn could be very fruitful for enhancing the ultimate accuracy [3].

Vas_Pat_Thin_Algo()

File $* \mathrm{fb}, * \mathrm{ft}$;

*fb=fopen("bin.jpeg",,"r");

*ft=fopen(“thin.jpeg","w”);

intmatsrc[100][100], maddest[100][100],r,c,i,j;

matsrc[][] $\square$ Pixel Intensity Value of fb;

$\mathrm{r} \square$ Image Width;

c $\square$ Image Height;

while(!EOF of fb)

\{

for $(\mathrm{i}=1 ; \mathrm{i}<\mathrm{r}-1 ; \mathrm{i}++)$

\{

for $(j=1 ; j<c-1 ; j++)$

\{

if $((\operatorname{matsrc}[\mathrm{i}][\mathrm{j}-1]==0$ \&\&matsrc $[\mathrm{i}][\mathrm{j}]==0 \quad \& \&$

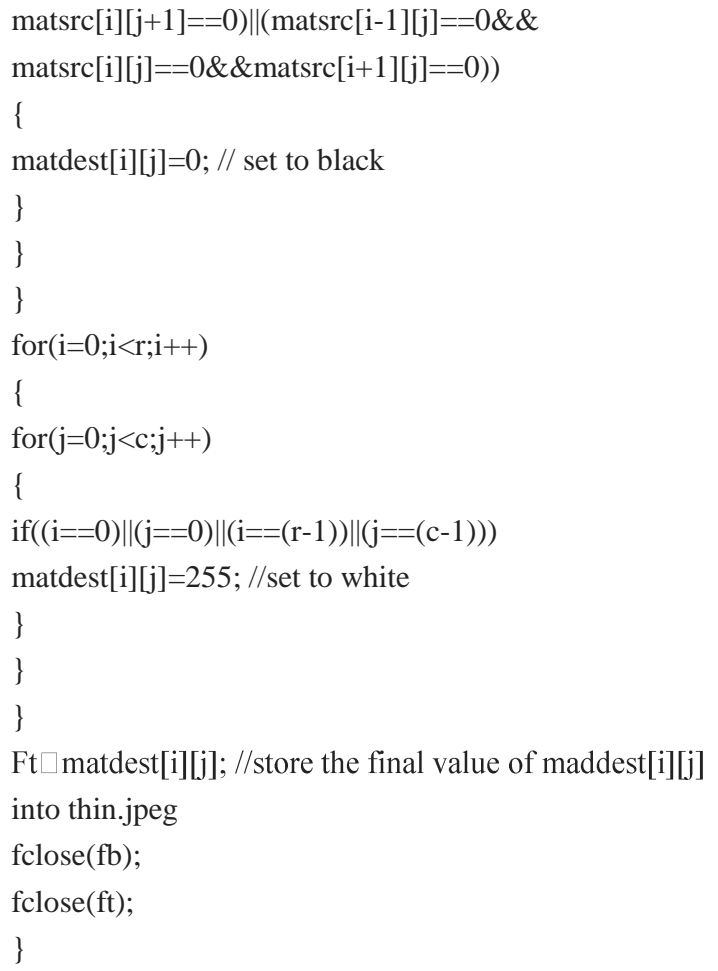

\section{FIGURES}

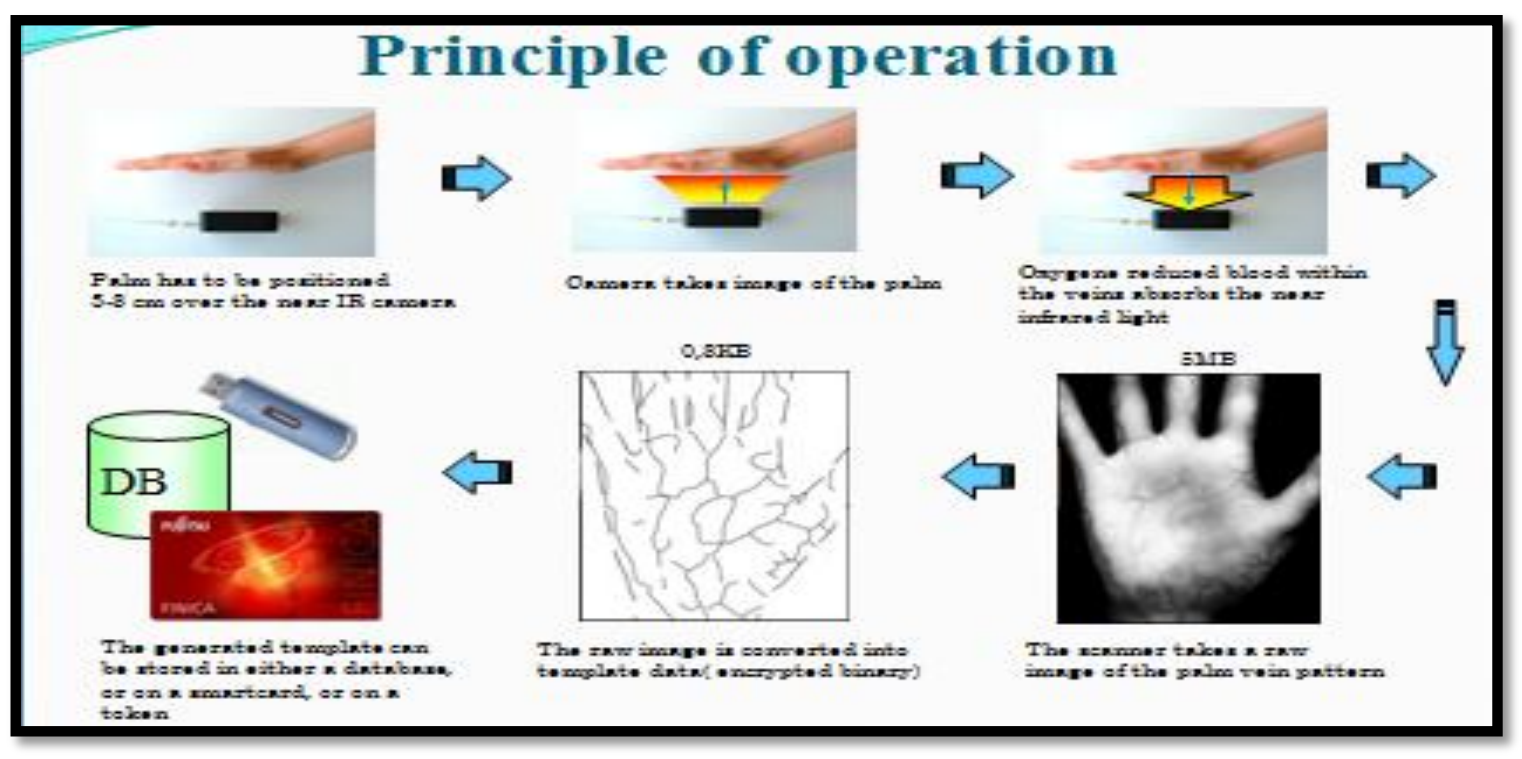

Fig 1: Principle of operation 


\section{RESULTS}

LOGIN FORM:

UPLOADING PALM IMAGE:
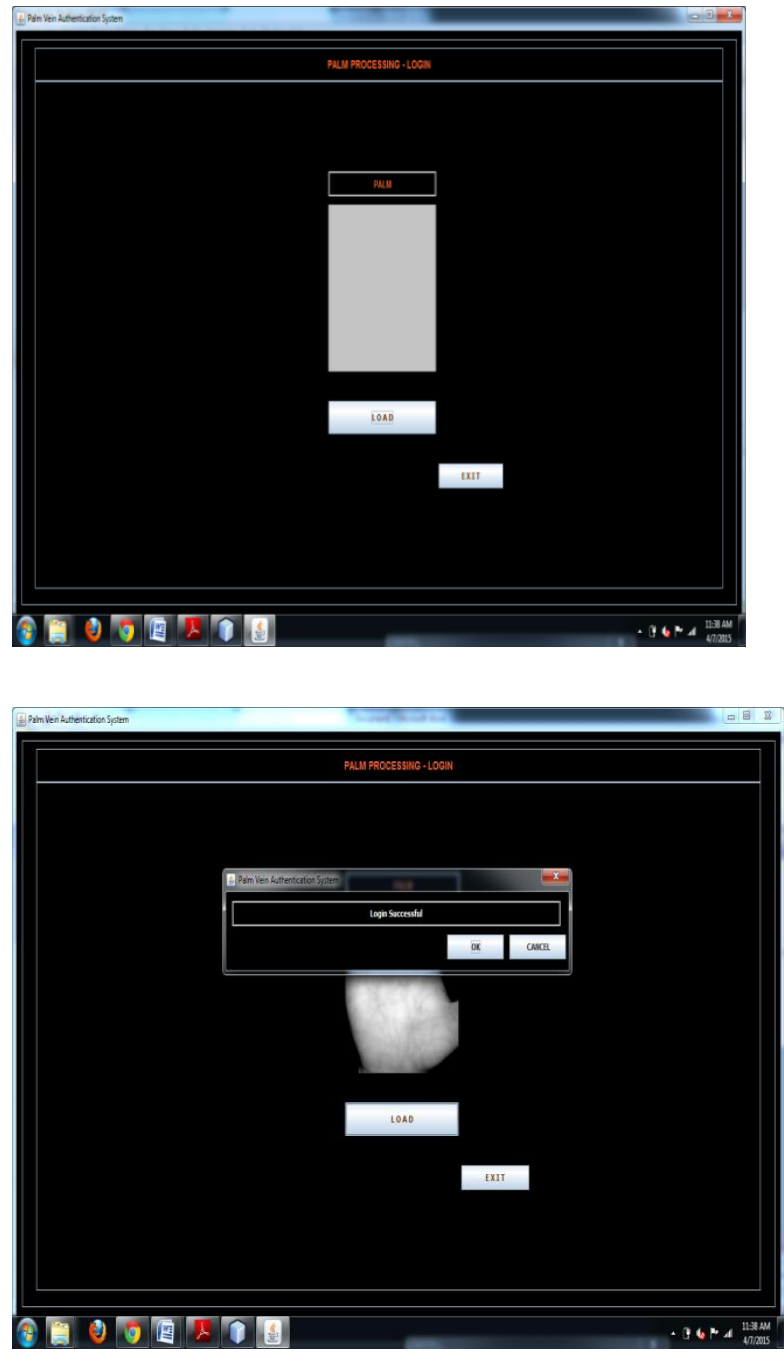

MAIN FORM:

ROI EXTRACTION STEP 1:

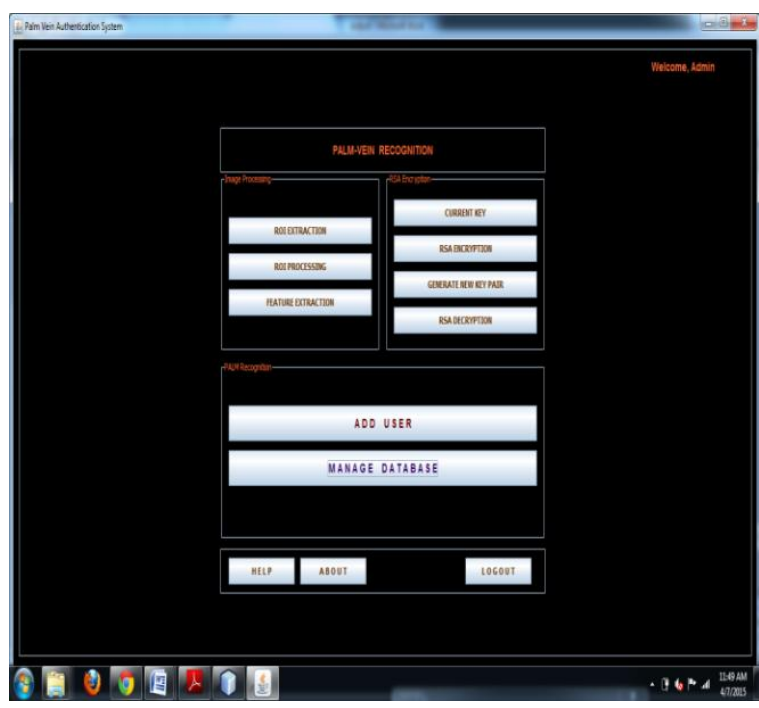

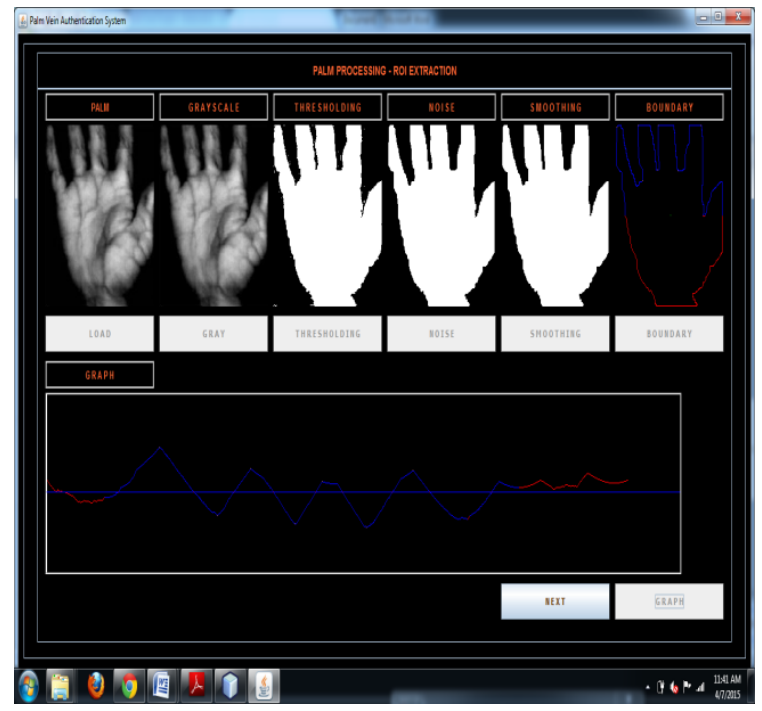

ROI EXTRACTION STEP 2:

ROI EXTRACTION STEP 3:
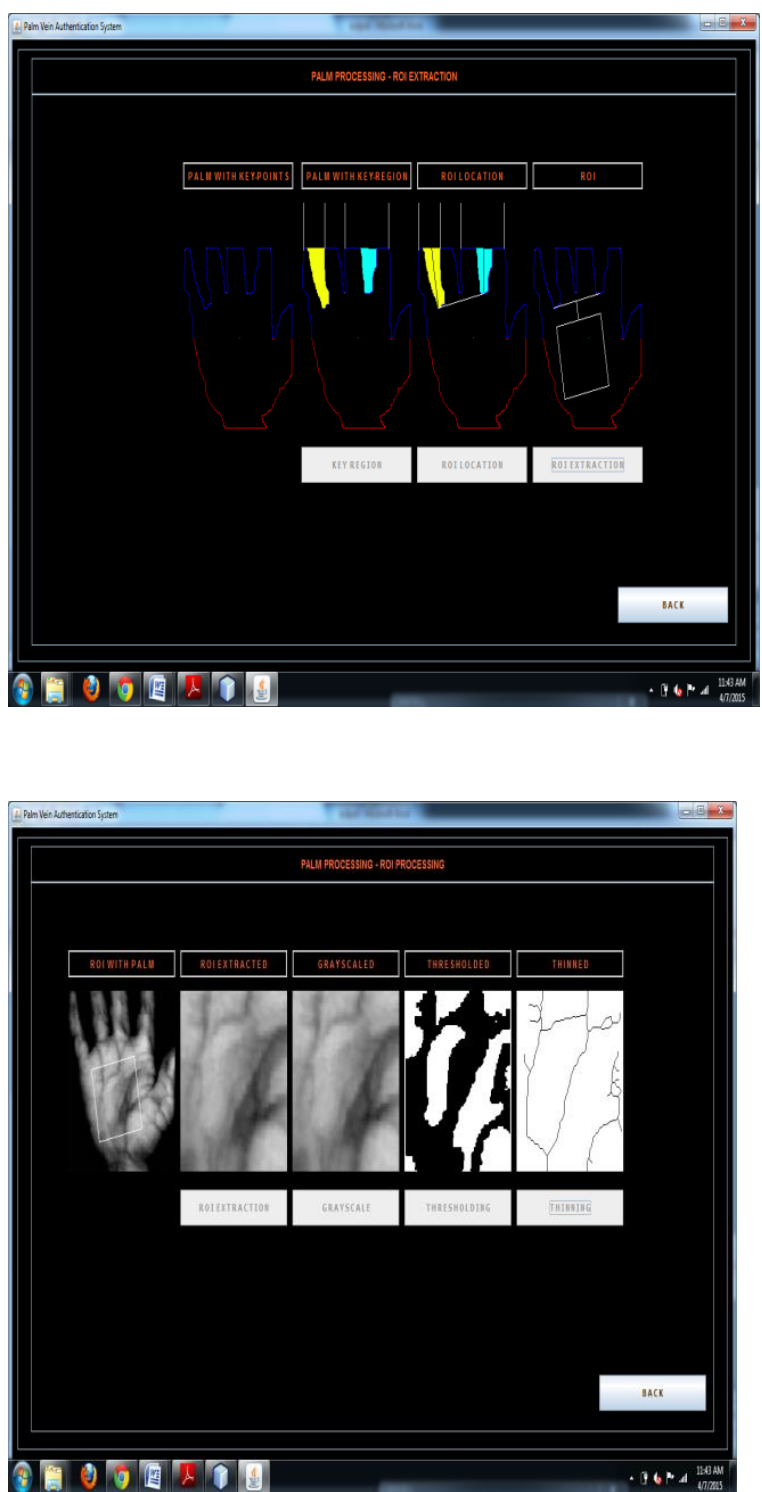
FEATURE EXTRACTION:

DISPLAYING CURRENT KEY:
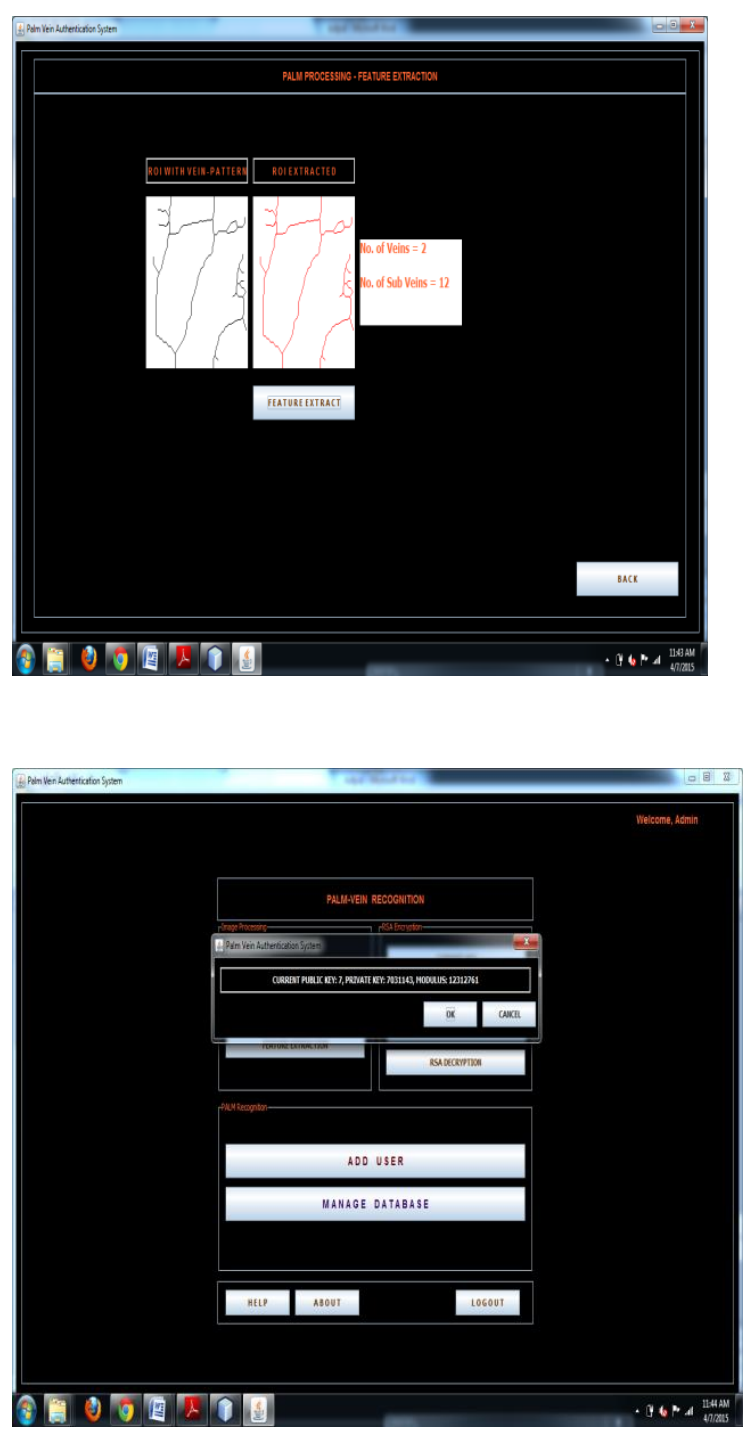

ENCRYPTING IMAGE FILE:

GENERATING KEY:

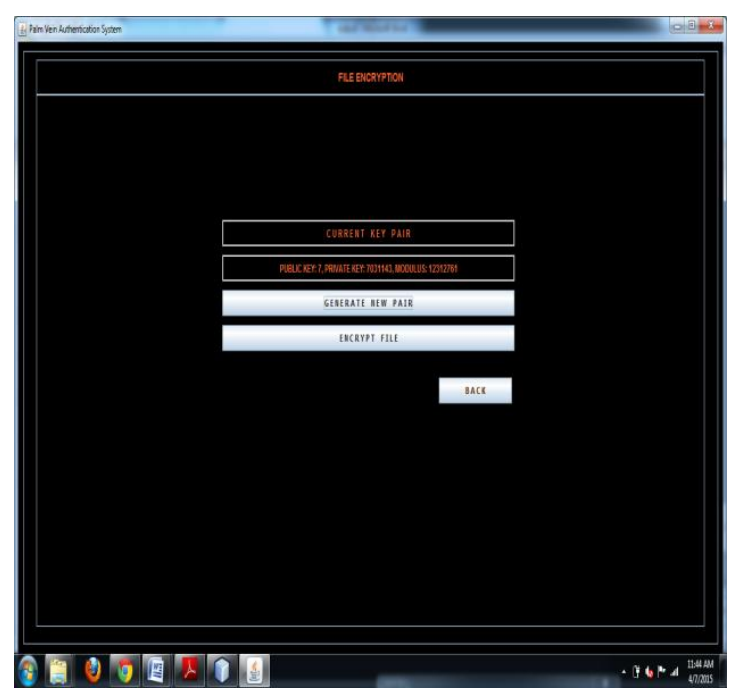

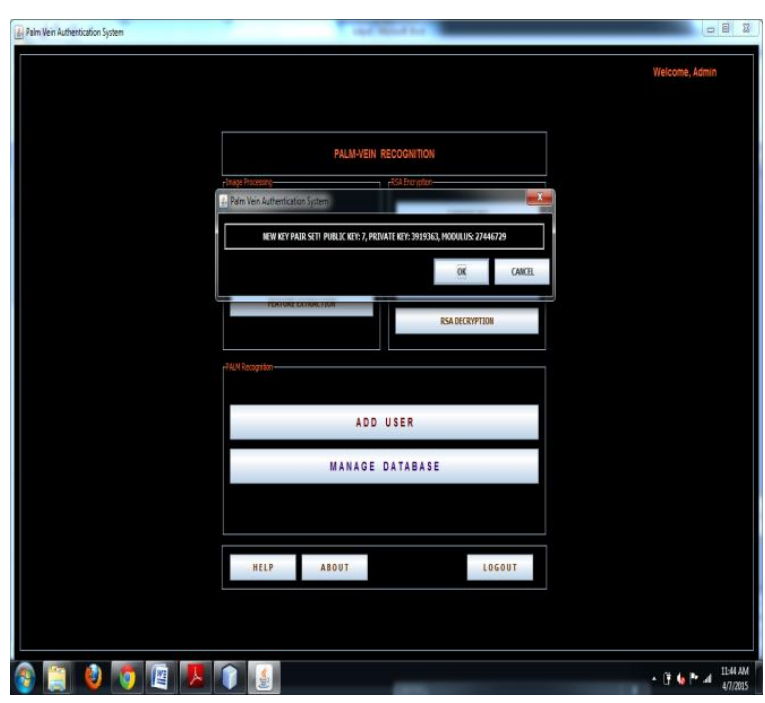

DECRYPTING IMAGE FILE:

ADDING USER TO DATABASE:
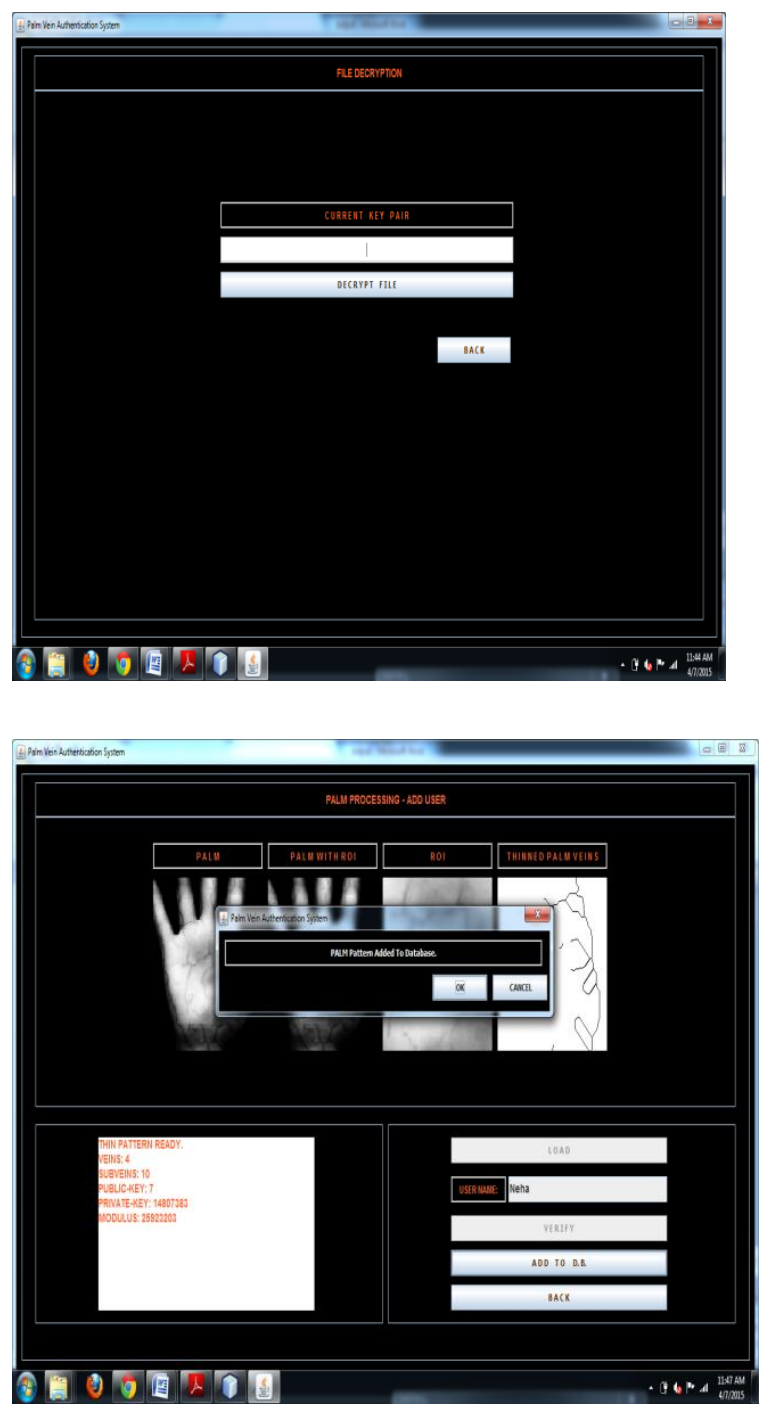

MANAGING DATABASE: 


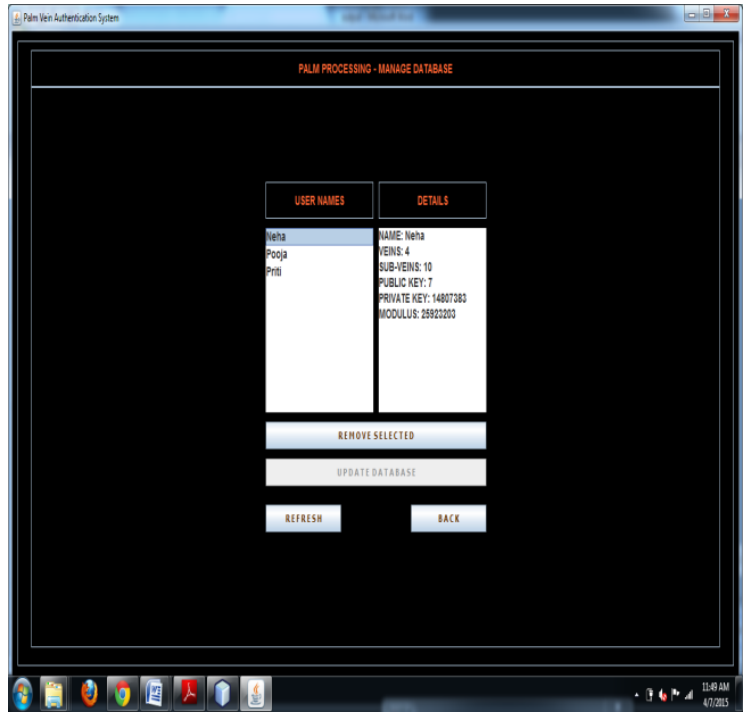

\section{CONCLUSION}

The objective of the project is Palm Vein authentication is the process of identifying a person on the basis of palm vein template. Palm Veins are identified by comparing palm veins image stored in the database. User identification is basically a pattern classification problem preceded by a feature extraction stage. The future scope of this project is, it can be used using a less expensive infrared camera so that it can be applicable everywhere in Banks, ATMs, and Colleges as a biometric system.

\section{ACKNOWLEDGMENTS}

The author wishes to thank contributors of the previous research work and we also thank our family members for their support throughout.

\section{REFERENCES}

[1] Mansi Manocha and Parminder Kaur, "Palm Vein Recognition for Human Identification Using NN", International Journal of Innovative Technology and Exploring Engineering(IJITEE)

[2] Junichi Hashimoto, "Finger Vein Authentication Technology and its Future", 2006 Symposium on VLSI
Circuits Digest of Technical Papers, 2006, pp. 5-8. [Jain, 04] Anil K. Jain, Arun Ross and SalilPrabhakar, "An Introduction to Biometric Recognition", IEEE Transactions on Circuits and Systems for Video Technology, Vol. 14, No. 1, January 2004, pp. 4-20.

[3] P. Ghosh and R. Dutta, "A new approach towards Biometric Authentication System in Palm Vein Domain"

[4] David Mulyono, Horng Shi Jinn, "A Study of Finger Vein Biometric for Personal Identification", International Symposium on Biometrics and Security Technologies, 2008, 23-24 April, 2008, Islamabad, pp. 1-8.

[5] KornelijeRabuzin, Miroslav Baca and MirkoMalekovic, "A Multimodal Biometric System Implemented within an Active Database Management System", Journal of Software, Vol. 2, No. 4, October 2007, pp. 24-31.

[6] Toshiyuki Tanaka, Naohiko Kubo, "Biometric Authentication by Hand Vein Patterns", SICE Annual Conference, Sapporo, August 4-6, 2004, pp. 249-253.

[7] Shi Zhao, Yiding Wang and Yunhong Wang, "Extracting Hand Vein Patterns from Low-Quality Images: A New Biometric Technique Using Low-Cost Devices", Fourth International Conference on Image and Graphics, 2007, pp. 667-671.

[8] Mansi Manocha and Parminder Kaur, "Palm Vein Recognition for Human Identification Using NN", International Journal of Innovative Technology and Exploring Engineering(IJITEE)

[9] Ding, Yuhang Ding, Dayan Zhuang and Kejun Wang, "A Study of Hand Vein Recognition Method", The IEEE International Conference on Mechatronics \& Automation Niagara Falls, Canada, July 2005, pp. 2106-2110.

[10] Basics of Image Processing. http://www.visionsystem.com/technology/machinevision _overview.php

[11] Fujustu "Palm Vein Pattern Authentication Technology" White paper printed in the U.S.A. Qty. 3M WP10800306 